\title{
NOVO REATOR ELETRÔNICO COM ELEVADO FATOR DE POTÊNCIA PARA MÚLTIPLAS LÂMPADAS FLUORESCENTES TUBULARES
}

\author{
Fabio Toshiaki Wakabayashi \\ Universidade Estadual Paulista \\ UNESP - FEIS - DEE \\ Cx. Postal 31 - 15385-000 - Ilha Solteira (SP) \\ Fax: (18) 3742-2735 \\ e-mail: canesin@dee.feis.unesp.br
}

Carlos Alberto Canesin

\begin{abstract}
Resumo - Este artigo apresenta um novo reator eletrônico, com fator de potência e rendimento elevados, para múltiplas lâmpadas fluorescentes tubulares. O estágio de entrada deste reator é um novo retificador Sepic com comutação em corrente nula (ZCS) e modulação por largura de pulso (PWM). Utiliza-se a técnica de controle por valores médios de corrente para propiciar elevado fator de potência e atender às normas IEC 61000-3-2. Com relação ao estágio de saída, este é um clássico inversor ressonante Half-Bridge com comutação em tensão nula (ZVS). Desenvolve-se um exemplo de projeto do novo reator eletrônico, projetado para alimentar 5 lâmpadas fluorescentes 40W-T12, com $220 \mathrm{~V}$ de tensão eficaz de alimentação, $115 \mathrm{~V}$ de tensão média para o barramento de corrente contínua, com os estágios retificador e inversor operando em $50 \mathrm{kHz}$. Resultados experimentais são apresentados para validar a análise desenvolvida. A taxa de distorção harmônica (TDH) na corrente de entrada é igual a 7,59\% para uma TDH na tensão de alimentação igual a $1,56 \%$, em condições nominais. $O$ rendimento global medido é de cerca de $92,1 \%$ para carga nominal.
\end{abstract}

Abstract - This paper presents a novel electronic ballast, featuring high power-factor and high efficiency, for multiple tubular fluorescent lamps. The input stage of this ballast is a new Zero-Current-Switching (ZCS) Pulse-Width-Modulated (PWM) Sepic rectifier. The average-current control technique is used in order to provide high power-factor and to fit the input current into IEC 61000-3-2 standards. Regarding to the output stage, it is a classical resonant Half-Bridge inverter, performing Zero-Voltage-Switching (ZVS). It is developed a design example for the new electronic ballast, designed to feed five 40W-T12 fluorescent lamps, with $220 \mathrm{~V}_{\text {rms }}$ input voltage, $115 \mathrm{~V}_{\text {avg }}$ dc link voltage, and rectifying and inverting stages operating at $50 \mathrm{kHz}$. Finally, experimental results are presented in order to verify the developed analysis. The Total Harmonic Distortion (THD) at input current is equal to $7.59 \%$ for an input voltage THD equal to $1.56 \%$, at full load. The measured overall efficiency is about $92.1 \%$, at rated load.

\section{INTRODUÇÃO}

Atualmente, a crescente demanda mundial de energia elétrica tornou imprescindível a implementação de projetos de racionalização do consumo. Dentro deste contexto, sistemas de iluminação artificial constituem cargas bastante importantes a serem analisadas. No Brasil, estima-se que cerca de $17 \%$ do consumo de energia elétrica em ambientes residenciais e comerciais sejam advindos deste tipo de carga.

Em função da crise no setor elétrico brasileiro, tem-se incentivado o uso de sistemas de iluminação fluorescente, os quais apresentam elevada eficácia luminosa (lumens/Watt). Apesar disto, certos aspectos dos sistemas fluorescentes podem ser apontados como desvantagens.

De início, faz-se necessário o uso de um dispositivo denominado reator para iluminação, com a finalidade de controlar o fluxo de corrente através das lâmpadas fluorescentes, tendo em vista que estas apresentam característica de resistência negativa [1]. Tal fato encarece o custo de implementação deste sistema, em relação ao sistema incandescente. Em sua concepção mais simples, o dispositivo é denominado de reator eletromagnético e é composto por um autotransformador e associação de elementos reativos. Por ser operado na mesma freqüência da rede de alimentação em corrente alternada (CA), alguns problemas podem ser identificados, tais como: ruído audível, efeito estroboscópico, peso e volume elevados e reduzida eficiência.

Para minimizar tais problemas, aperfeiçoamentos vêm sendo constantemente propostos. A operação em elevadas freqüências foi uma das grandes inovações incorporadas aos reatores, permitindo a supressão de ruídos audíveis e do efeito estroboscópico, além da redução de peso e volume da estrutura. Uma outra vantagem da operação em elevadas freqüências é o aumento do fluxo luminoso da lâmpada fluorescente [1], em relação àquele advindo de uma lâmpada fluorescente alimentada em reduzidas freqüências, para uma mesma potência processada.

Para que as lâmpadas fluorescentes pudessem ser operadas em elevadas freqüências, um novo dispositivo denominado de reator eletrônico foi desenvolvido. Este, por sua vez, é geralmente composto por um estágio de entrada retificador acoplado a um estágio inversor, o qual opera em elevadas freqüências. Entretanto, elevadas freqüências de operação podem causar significativas perdas durante o processo de comutação dos semicondutores. Em função disto, estágios inversores que incorporam técnicas de comutação nãodissipativa têm sido usados. Quanto ao retificador de entrada, este é geralmente formado por uma ponte de diodos e um filtro capacitivo de elevado valor. Assim, a defasagem angular e a distorção harmônica impostas à corrente de entrada do reator são significativas, implicando num fator de 
potência bastante reduzido. Portanto, para que os reatores eletrônicos tornem-se mais eficientes, é necessária a utilização de estágios retificadores que incorporem técnicas de correção do fator de potência [2].

Por fim, quando comparado ao sistema de iluminação incandescente, o custo associado à implementação do sistema fluorescente é geralmente apontado como sendo sua maior desvantagem. Entretanto, a elevada eficiência luminosa associada à maior durabilidade das lâmpadas fluorescentes faz com que os custos iniciais sejam amortizados, em uma análise de médio a longo prazos, tornando vantajoso tal investimento. Mesmo assim, com o intuito de aumentar a atratividade destes sistemas de iluminação, o conceito de reatores eletrônicos capazes de operar múltiplas lâmpadas fluorescentes foi proposto [3], visando a redução dos custos de implementação associados a este sistema.

Desta forma, este artigo apresenta um novo reator eletrônico com elevado fator de potência, destinado à operação de múltiplas lâmpadas fluorescentes tubulares. O estágio de entrada deste reator é um novo retificador Sepic com comutação em corrente nula (ZCS) e modulação por largura de pulso (PWM), controlado através da técnica de valores médios instantâneos de corrente. Já o estágio de saída do reator é composto por um clássico inversor Half-Bridge ressonante, o qual incorpora comutação em tensão nula (ZVS) em seus dispositivos semicondutores, controlado por um regulador de baixo custo, o IR2155, adaptado para alimentação de cinco lâmpadas fluorescentes 40W-T12.

\section{O NOVO REATOR ELETRÔNICO COM ELEVADO FATOR DE POTÊNCIA}

A Figura 1 mostra o novo reator eletrônico proposto.

O estágio retificador Sepic ZCS-PWM incorpora uma nova célula de comutação derivada de [4], na qual os interruptores ativos $S_{1}$ e $S_{2}$ são acionados de forma ZCS e bloqueados em corrente e tensão nulas (ZCZVS), enquanto que os diodos $\mathrm{D}_{1}$ e $\mathrm{D}_{2}$ apresentam entrada em condução do tipo ZVS. O estágio inversor é um conversor Half-Bridge acoplado a filtros ressonantes $\left(\mathrm{L}_{\mathrm{s}(\mathrm{n})}, \mathrm{C}_{\mathrm{s}(\mathrm{n})}\right.$ e $\left.\mathrm{C}_{\mathrm{p}(\mathrm{n})}\right)$. Informa-se ainda que os interruptores $S_{3}$ e $S_{4}$ apresentam entrada em condução do tipo ZVS.

A análise deste novo reator eletrônico pode ser realizada com a apresentação individual de seus dois estágios, uma vez que ambos são operados de forma independente.

\section{A. O Novo Retificador Sepic ZCS-PWM com Elevado Fator de Potência}

A análise desta nova estrutura é desenvolvida com base nas seguintes considerações simplificadoras: todos os componentes são ideais; a tensão de alimentação é considerada praticamente constante $\left(\mathrm{V}_{\text {in }}\left(\omega \mathrm{T}_{\mathrm{i}}\right)\right)$ durante um período genérico de chaveamento $\left(\mathrm{T}_{\mathrm{i}}=\mathrm{T}_{\text {Sepic }}\right)$, pois a freqüência de operação do conversor $\left(\mathrm{f}_{\text {Sepic }}\right)$ é muito maior do que a freqüência da rede de $\mathrm{CA}\left(\mathrm{f}_{\mathrm{CA}}\right)$.

$\mathrm{O}$ filtro de entrada $\left(\mathrm{L}_{\mathrm{in}}\right)$ associado à ponte de diodos $\left(\mathrm{D}_{\mathrm{r} 1} \mathrm{a}\right.$ $\mathrm{D}_{\mathrm{r} 4}$ ) é substituído por uma fonte de corrente retificada $\left(\left|\mathrm{I}_{\text {in }}(\omega \mathrm{t})\right|\right)$, e seu valor é assumido constante $\left(\left|\mathrm{I}_{\mathrm{in}}\left(\omega \mathrm{T}_{\mathrm{i}}\right)\right|\right)$ durante um período genérico de chaveamento $\left(\mathrm{T}_{\mathrm{i}}\right)$; a indutância de acumulação $\left(\mathrm{L}_{\mathrm{M}}\right)$ é suficientemente elevada para ser assumida como uma fonte de corrente constante $\left(\mathrm{I}_{\mathrm{M}}\left(\omega \mathrm{T}_{\mathrm{i}}\right)\right)$, cujo valor pode ser definido a partir da equação (1), durante um período genérico de chaveamento.

$$
I_{M}\left(\omega T_{i}\right)=\frac{\pi}{2} \cdot I_{o(n o m)} \cdot\left|\operatorname{sen}\left(\omega T_{i}\right)\right|
$$

sendo: $\mathrm{I}_{\mathrm{o}(\mathrm{nom})}=$ valor médio nominal da corrente de saída do estágio retificador; a capacitância de acumulação $\left(\mathrm{C}_{\mathrm{e}}\right)$ é elevada o bastante para ser considerada uma fonte de tensão constante $\left(\mathrm{V}_{\mathrm{Ce}}\left(\omega \mathrm{T}_{\mathrm{i}}\right)=\left|\mathrm{V}_{\mathrm{in}}\left(\omega \mathrm{T}_{\mathrm{i}}\right)\right|=\right.$ valor instantâneo da tensão de alimentação retificada), durante um período genérico de chaveamento $\left(\mathrm{T}_{\mathrm{i}}\right)$; a tensão de saída $\left(\mathrm{V}_{\mathrm{o}}\right)$ do estágio retificador é constante.

\section{1) Etapas de funcionamento}

A Figura 2 mostra as principais formas de ondas idealizadas em conjunto com as etapas de funcionamento do retificador proposto, durante um período genérico de chaveamento.

Pode-se notar, com base na Figura 2.a, que o interruptor principal $\mathrm{S}_{1}$ é acionado em ZCS, em $\mathrm{t}=\mathrm{t}_{0}$, o mesmo ocorrendo com o interruptor auxiliar $\mathrm{S}_{2}$, em $\mathrm{t}=\mathrm{t}_{2}$. Além disso, ambos são bloqueados simultaneamente, no decorrer da sexta etapa de funcionamento $\left(\Delta \mathrm{t}_{6}=\mathrm{t}_{6}-\mathrm{t}_{5}\right)$, em ZCZVS. Informa-se que os diodos $\mathrm{D}_{1}$ e $\mathrm{D}_{2}$ são levados à condução de forma ZVS, em $\mathrm{t}=\mathrm{t}_{3}$ e $\mathrm{t}=\mathrm{t}_{8}$, respectivamente. Adicionalmente, informa-se que nesta célula de comutação proposta, o diodo $\mathrm{D}_{1}$ não conduz em conjunto como diodo $\mathrm{D}_{2}$, deficiência apresentada nas concepções anteriores desta célula [4 e 5], permitindo o aumento do rendimento da estrutura.

\section{2) Condições para obtenção de comutação ZCS}

Com o intuito de se garantir a obtenção de comutação ZCS para os interruptores ativos $\mathrm{S}_{1}$ e $\mathrm{S}_{2}$, conforme descrito anteriormente, é necessário que as restrições impostas pelas inequações (2) e (3) sejam conjuntamente satisfeitas.

$$
\beta=\frac{\mathrm{L}_{\mathrm{r} 2}}{\mathrm{~L}_{\mathrm{r} 1}}<1
$$

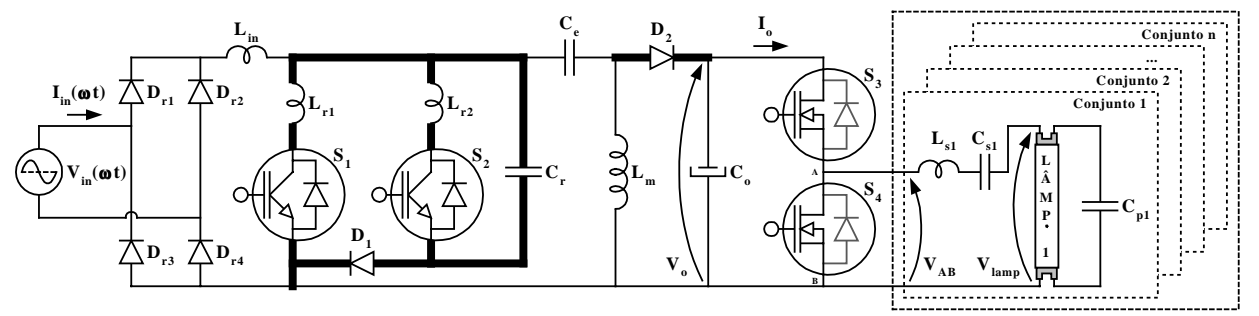

Figura 1 - Novo reator eletrônico com elevado fator de potência para múltiplas lâmpadas fluorescentes. 


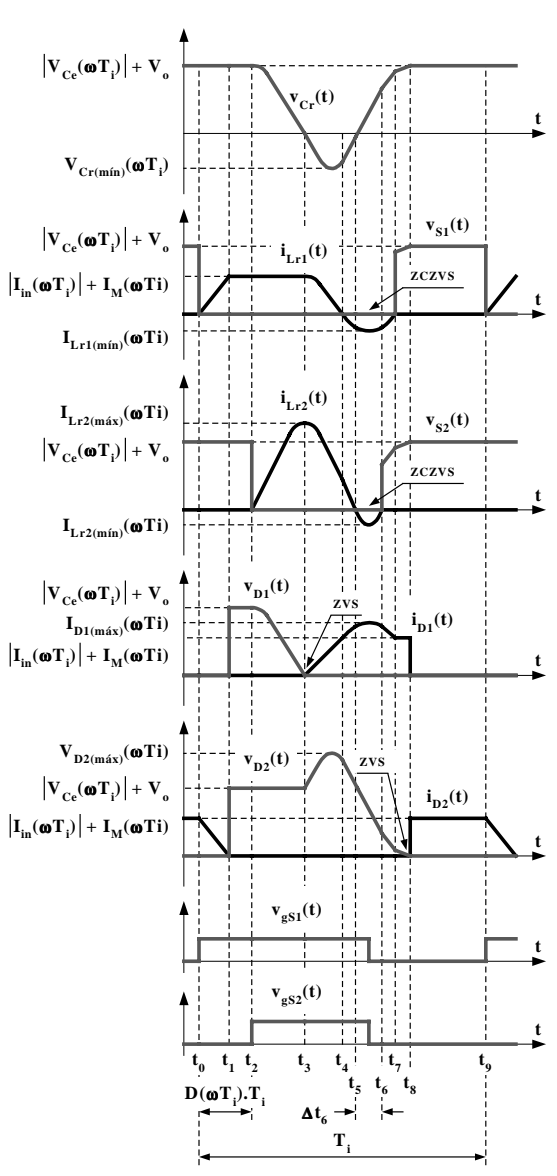

(a)

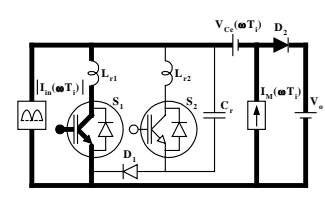

$\left(1^{\underline{a}}\right)\left[\mathbf{t}_{0}, \mathbf{t}_{1}\right]$

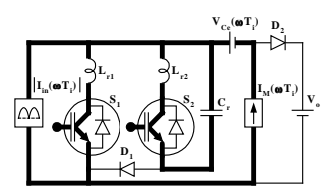

$\left(3^{\mathbf{a}}\right)\left[\mathbf{t}_{2}, \mathbf{t}_{3}\right]$

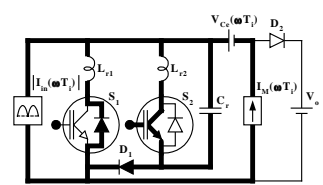

$\left(5^{-}\right)\left[t_{4}, t_{5}\right]$

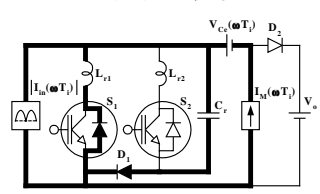

$\left(7^{\mathrm{a}}\right)\left[\mathbf{t}_{6}, \mathbf{t}_{7}\right]$

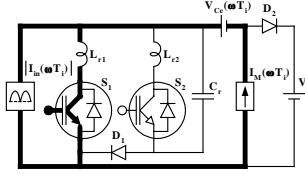

$\left(2^{\underline{a}}\right)\left[\mathbf{t}_{1}, \mathbf{t}_{2}\right]$

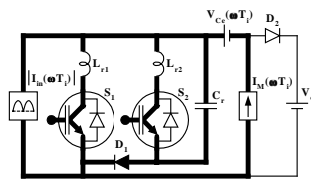

$\left(4^{\mathrm{a}}\right)\left[\mathrm{t}_{3}, \mathrm{t}_{4}\right]$

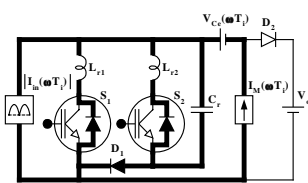

$\left(6^{\mathbf{a}}\right)\left[\mathbf{t}_{5}, \mathbf{t}_{6}\right]$

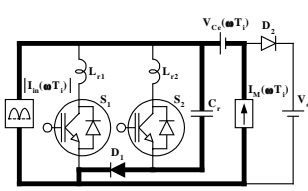

$\left(8^{\mathbf{a}}\right)\left[\mathbf{t}_{7}, \mathbf{t}_{8}\right]$

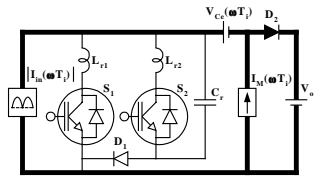

$\left(\mathbf{9}^{\mathbf{a}}\right)\left[\mathbf{t}_{8}, \mathbf{t}_{9}\right]$

Figura 2 - (a) Principais formas de ondas idealizadas, e (b) Etapas de funcionamento do novo retificador Sepic ZCS-PWM com elevado fator de potência, durante um período genérico de chaveamento $\left(\mathrm{T}_{\mathrm{i}}\right)$.

$$
\alpha_{\text {máx }}=\frac{\mathrm{I}_{\text {in }(\mathrm{p})}+\frac{\pi}{2} \cdot \mathrm{I}_{\mathrm{o}}}{\mathrm{V}_{\text {in }(\mathrm{p})}+\mathrm{V}_{\mathrm{o}}} \cdot \sqrt{\frac{\mathrm{L}_{\mathrm{r} 2}}{\mathrm{C}_{\mathrm{r}}}}<\beta
$$

sendo: $\quad I_{\text {in(p) }}=$ valor de pico da corrente de entrada;

$\mathrm{V}_{\text {in(p) }}=$ valor de pico da tensão de alimentação.

$\mathrm{O}$ intervalo de tempo disponível para o bloqueio simultâneo dos interruptores $S_{1}$ e $S_{2}$ é obtido através de (4).

$$
\Delta \mathrm{t}_{\mathrm{off}}=\frac{\Delta \mathrm{t}_{6}}{2}=\frac{\sqrt{1+\beta}}{\omega_{\mathrm{r} 2}} \cdot\left(\frac{\pi-\arccos (-\beta)}{(1+\beta)}\right)
$$

sendo:

$$
\omega_{\mathrm{r} 2}=\frac{1}{\sqrt{\mathrm{L}_{\mathrm{r} 2} \cdot \mathrm{C}_{\mathrm{r}}}}
$$

O ganho estático (q) do novo retificador Sepic ZCS-PWM é definido conforme [4], e é dado pela equação (6).

$$
\mathrm{q}\left(\alpha_{(\mathrm{ef})}, \beta, \mathrm{f}, \mathrm{D}_{(\mathrm{ef})}\right)=\frac{\mathrm{V}_{\mathrm{o}}}{\mathrm{V}_{\text {in (ef })}}=\frac{\mathrm{F}\left(\alpha_{(\mathrm{ef})}, \beta, \mathrm{f}, \mathrm{D}_{(\mathrm{ef})}\right)}{1-\mathrm{F}\left(\alpha_{(\mathrm{ef})}, \beta, \mathrm{f}, \mathrm{D}_{(\mathrm{ef})}\right)}
$$

sendo:

$$
\begin{gathered}
\alpha_{(\mathrm{ef})}=\frac{\mathrm{I}_{\text {in (ef })}+\mathrm{I}_{\mathrm{M}(\mathrm{ef})}}{\mathrm{V}_{\text {in (ef })}+\mathrm{V}_{\mathrm{o}}} \cdot \sqrt{\frac{\mathrm{L}_{\mathrm{r} 2}}{\mathrm{C}_{\mathrm{r}}}} \\
\mathrm{f}=\frac{2 \cdot \pi \cdot \mathrm{f}_{\text {Sepic }}}{\omega_{\mathrm{r} 2}}
\end{gathered}
$$

$\mathrm{D}_{(\mathrm{ef})}=$ razão cíclica eficaz de $\mathrm{S}_{1}$;

$I_{\text {in }(e f)}=$ valor eficaz da corrente de entrada;

$\mathrm{I}_{\mathrm{M}(\mathrm{ef})}=$ valor eficaz da corrente através de $\mathrm{L}_{\mathrm{M}}$;
$V_{\text {in(ef) }}=$ valor eficaz da tensão de alimentação.

A Figura 3 mostra o ganho estático do novo retificador Sepic como uma função de $\alpha_{(\text {ef })}$, ou seja, em relação à variação de carga, para diferentes valores de $\beta$ e f, tomandose $\mathrm{D}_{(\mathrm{ef})}$ como parâmetro de controle.

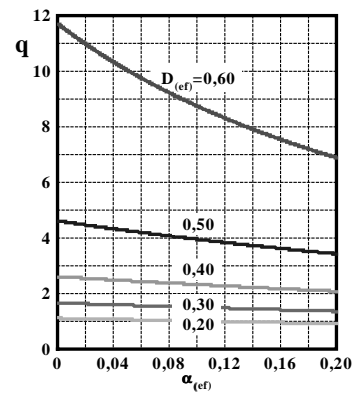

(a) $\beta=0,2 ; f=0,30$

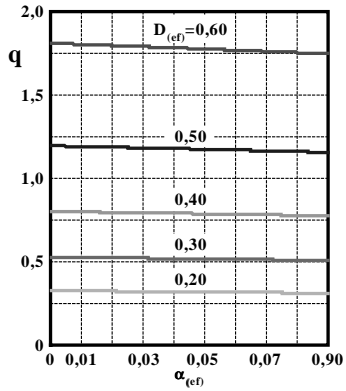

(b) $\beta=0,9 ; \mathrm{f}=0,05$
Figura 3 - Curvas de ganho estático para o novo retificador Sepic ZCS-PWM com elevado fator de potência.

Nota-se que valores elevados de $\beta$ associados a reduzidos valores de f propiciam boa regulação da tensão de saída. Contudo, valores de $\beta$ próximos à unidade podem implicar em indutores ressonantes de volume significativo. Além disso, valores reduzidos de $\mathrm{f}$ podem conduzir a elevadas freqüências de ressonância, resultando em perdas mais acentuadas nos elementos magnéticos, além de problemas de 
interferência eletromagnética. Assim, devem ser adotados valores para $\beta$ e f que proporcionem a obtenção de reduzida influência da ressonância sobre a regulação da tensão de saída e que evitem a ocorrência dos problemas citados.

\section{B. O Inversor Ressonante Half-Bridge}

A alimentação em elevadas freqüências $(>5 \mathrm{kHz})$ faz com que as lâmpadas fluorescentes apresentem uma característica dinâmica similar à de uma carga resistiva [6]. Então, pode-se utilizar uma resistência equivalente $\left(\mathrm{R}_{\text {lamp }}\right)$ no lugar da lâmpada para simplificação da análise do circuito, sem perda significativa de precisão. Nesta topologia, os interruptores ativos entrarão em condução de forma ZVS caso a freqüência de chaveamento do estágio inversor $\left(\mathrm{f}_{\mathrm{HB}}\right)$ seja superior à freqüência de ressonância do ramo série $\left(\mathrm{f}_{\mathrm{rs}}\right)$, sendo:

$$
\begin{aligned}
& \mathrm{f}_{\mathrm{rs}}=\frac{1}{2 \cdot \pi \cdot \sqrt{\mathrm{L}_{\mathrm{s}} \cdot \mathrm{C}_{\mathrm{s}}}} ; \\
& \mathrm{e}: \quad \mathrm{L}_{\mathrm{s}}=\mathrm{L}_{\mathrm{s} 1}=\mathrm{L}_{\mathrm{s} 2}=\ldots=\mathrm{L}_{\mathrm{s}(\mathrm{n})} ; \\
& \mathrm{C}_{\mathrm{s}}=\mathrm{C}_{\mathrm{s} 1}=\mathrm{C}_{\mathrm{s} 2}=\ldots=\mathrm{C}_{\mathrm{s}(\mathrm{n})} .
\end{aligned}
$$

\section{1) Etapas de funcionamento}

A análise deste inversor é desenvolvida considerando-se apenas uma lâmpada fluorescente. Entretanto, a extensão desta análise para um conjunto de lâmpadas é bastante simples, uma vez que a seqüência de etapas de funcionamento é preservada. A Figura 4 mostra as principais formas de onda idealizadas em conjunto com as quatro etapas de funcionamento do inversor, para um período de operação $\left(\mathrm{T}_{\mathrm{HB}}\right)$ do inversor ressonante Half-Bridge.

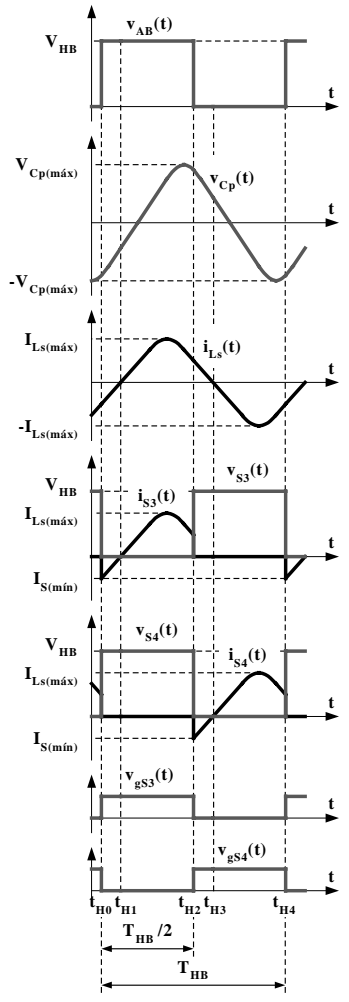

(a)

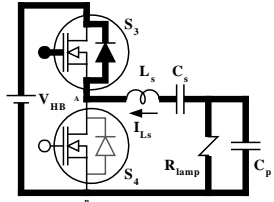

$\left(1^{\underline{a}}\right)\left[t_{H 0}, t_{H 1}\right]$

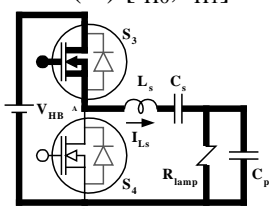

$\left(2^{\mathrm{a}}\right)\left[\mathrm{t}_{\mathrm{H} 1}, \mathrm{t}_{\mathrm{H} 2}\right]$

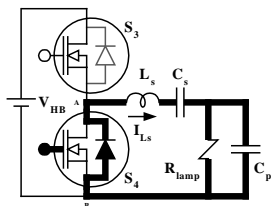

$\left(3^{\mathrm{a}}\right)\left[\mathrm{t}_{\mathrm{H} 2}, \mathrm{t}_{\mathrm{H} 3}\right]$

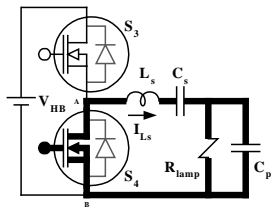

$\left(4^{\mathrm{a}}\right)\left[\mathrm{t}_{\mathrm{H} 3}, \mathrm{t}_{\mathrm{H} 4}\right]$ (b)
Figura 4 - (a) Principais formas de ondas idealizadas, e (b) Etapas de funcionamento do inversor Half-Bridge, para um período de chaveamento.
Os interruptores $\mathrm{S}_{3}$ e $\mathrm{S}_{4}$ são operados de forma complementar. Com base na Figura 4, nota-se que $S_{3}$ é acionado em ZVS em $\mathrm{t}=\mathrm{t}_{\mathrm{H} 0}$, tendo em vista que a corrente através de $\mathrm{L}_{\mathrm{S}}\left(\mathrm{i}_{\mathrm{Ls}}(\mathrm{t})\right)$ flui através do diodo em antiparalelo de $\mathrm{S}_{3}$, devido ao sentido imposto pelo circuito ressonante. De forma análoga, no início da segunda metade do período de operação, em $\mathrm{t}=\mathrm{t}_{\mathrm{H} 2}, \mathrm{~S}_{4}$ é acionado em ZVS.

\section{2) Processo de ignição da lâmpada fluorescente}

Para a ignição da lâmpada fluorescente, o reator deve impor um elevado nível de tensão através da mesma para a ocorrência do primeiro arco através da coluna de gás [1]. No entanto, para evitar danos significativos nos eletrodos da lâmpada, os mesmos deverão ser adequadamente aquecidos antes do início da ocorrência das descargas elétricas.

\section{1) Tensão de ignição da lâmpada fluorescente}

Antes da ocorrência do primeiro arco, o circuito inversor ressonante pode ser representado conforme a Figura 5.

Neste circuito, elevados valores de tensão através da lâmpada podem ser obtidos definindo-se a freqüência de chaveamento do estágio inversor $\left(\mathrm{f}_{\mathrm{HB}}\right)$ como sendo igual, ou próxima, à freqüência de ressonância do circuito $\left(\mathrm{f}_{\mathrm{rp}}\right)$, sendo:

$$
\mathrm{f}_{\mathrm{rp}}=\frac{1}{2 . \pi \cdot \sqrt{\mathrm{L}_{\mathrm{s}} \cdot \frac{\mathrm{C}_{\mathrm{s}} \cdot \mathrm{C}_{\mathrm{p}}}{\mathrm{C}_{\mathrm{s}}+\mathrm{C}_{\mathrm{p}}}}} ;
$$

e:

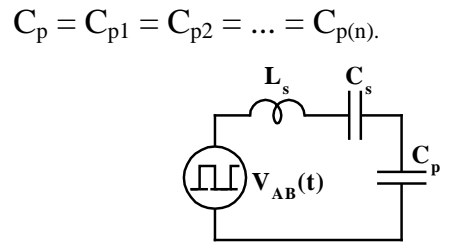

Figura 5 - Circuito equivalente para análise do processo de ignição

A Figura 6 mostra a forma de onda da tensão sobre a lâmpada quando as freqüências são iguais e quando as freqüências são ligeiramente diferentes.

É importante notar que para $\mathrm{f}_{\mathrm{HB}}=\mathrm{f}_{\mathrm{rp}}$, se por um motivo qualquer a descarga através da lâmpada não ocorrer, a tensão sobre a mesma pode assumir valores extremamente elevados, havendo então a possibilidade de danos aos componentes do reator. Por outro lado, caso $f_{H B}>f_{r p}$, verifica-se que o valor máximo da tensão pode ser limitado em função da ocorrência do fenômeno conhecido como "batimento" [3, 7 e 8], evitando-se possíveis danos ao reator em caso de falha no processo de ignição.

\section{2) Processo de preaquecimento dos eletrodos}

Da análise da Figura 6, é possível verificar que quanto maior for a diferença entre $f_{H B}$ e $f_{r p}$, menor será o valor da tensão máxima sobre a lâmpada. Assim, através do controle adequado da frequiência de chaveamento durante o processo de ignição, torna-se possível estabelecer um procedimento de aumento do tempo necessário à evolução da tensão a níveis suficientes para a ignição da lâmpada, fornecendo condições para um adequado processo de preaquecimento dos eletrodos. Para prover a desejada variação de freqüência de operação, será utilizada a técnica de chaveamento de capacitores em paralelo [8 e 9]. 


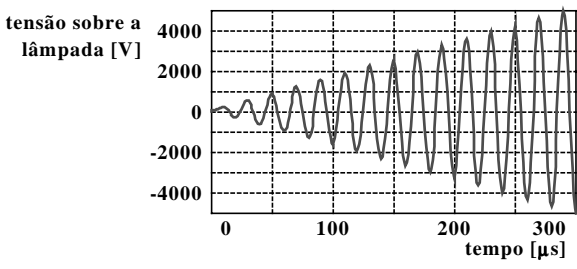

(a) $\mathrm{f}_{\mathrm{HB}}=\mathrm{f}_{\mathrm{rp}}$

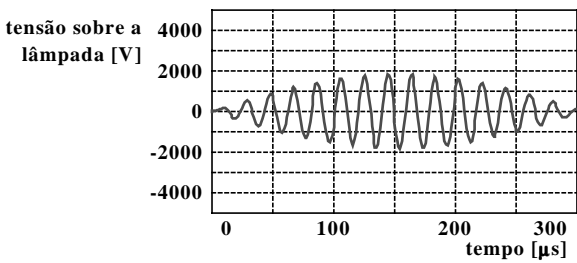

(b) $\mathrm{f}_{\mathrm{HB}}=1,065 . \mathrm{f}_{\mathrm{rp}}$

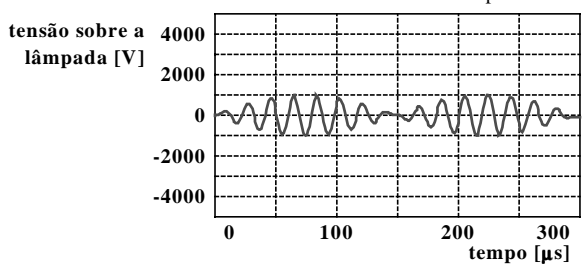

(c) $\mathrm{f}_{\mathrm{HB}}=1,135 . \mathrm{f}_{\mathrm{rp}}$

Figura 6 - Formas de onda da tensão sobre a lâmpada durante o processo de ignição, para diferentes valores de frequiência de chaveamento $\left(\mathrm{f}_{\mathrm{HB}}\right)$.

O circuito integrado IR2155 é usado para acionar adequadamente os interruptores $\mathrm{S}_{3}$ e $\mathrm{S}_{4}$ do estágio inversor Half-Bridge. A Figura 7 mostra um detalhe do diagrama esquemático do circuito de controle implementado.

De acordo com [10], enquanto $\mathrm{Q}_{\mathrm{T}}$ e $\mathrm{D}_{\mathrm{T}}$ estão bloqueados, a freqüência de chaveamento de $S_{3}$ e $S_{4}\left(f_{\mathrm{HB}(\mathrm{ph})}\right)$ será definida pela equação (11). Neste caso, como uma imposição da metodologia de projeto, o valor de $\mathrm{f}_{\mathrm{HB}(\mathrm{ph})}$ deverá ser bastante superior ao valor de $f_{\mathrm{HB}}$, permitindo a evolução do preaquecimento em função da limitação dos valores máximos de tensão sobre a lâmpada.

$$
\mathrm{f}_{\mathrm{HB}(\mathrm{ph})}=\frac{1}{1,4 \cdot\left(\mathrm{R}_{\mathrm{T}}+150\right) \cdot \mathrm{C}_{\mathrm{T} 1}} ;
$$

O tempo de preaquecimento é definido pela constante de tempo dada por $\mathrm{R}_{1}$ e $\mathrm{C}_{1}$. Quando o transistor $\mathrm{Q}_{\mathrm{T}}$ e o diodo $\mathrm{D}_{\mathrm{T}}$ entram em condução, os mesmos provêem, respectivamente, os caminhos para carga e descarga de $\mathrm{C}_{\mathrm{T} 2}$, caracterizando uma associação em paralelo entre $\mathrm{C}_{\mathrm{T} 1}$ e $\mathrm{C}_{\mathrm{T} 2}$. A freqüência de chaveamento passa então a ser definida pela equação (12).

$$
\mathrm{f}_{\mathrm{HB}}=\frac{1}{1,4 \cdot\left(\mathrm{R}_{\mathrm{T}}+150\right) \cdot\left(\mathrm{C}_{\mathrm{T} 1}+\mathrm{C}_{\mathrm{T} 2}\right)}
$$

Desta forma, de acordo com as equações (11) e (12), pode-se verificar que $\mathrm{f}_{\mathrm{HB}}<\mathrm{f}_{\mathrm{HB}(\mathrm{ph})}$.

3) Determinação dos elementos ressonantes do estágio inversor

Em função das considerações realizadas acerca das condições necessárias para garantir a obtenção de comutação ZVS e de ocorrência do "batimento", as restrições impostas pelas inequações (13) e (14) devem ser atendidas para que o circuito inversor seja adequadamente projetado.

$$
\begin{gathered}
f_{\text {ign }}=\frac{f_{H B}}{f_{r p}}>1 \\
f_{z v s}=\frac{f_{H B}}{f_{r s}}>\sqrt{\frac{f_{\text {ign }} \cdot V_{A B(e f)}}{V_{A B(e f)}+\left(f_{\text {ign }}^{2}-1\right) \cdot V_{\text {lamp (ef })}}}
\end{gathered}
$$

sendo: $\quad V_{\text {lamp(ef) }}=$ valor eficaz da tensão sobre a lâmpada;

$\mathrm{V}_{\mathrm{AB}(\mathrm{ef})}=$ valor eficaz da componente fundamental da tensão $\mathrm{V}_{\mathrm{AB}}$.

Então, de acordo com as definições anteriores, a determinação dos parâmetros do filtro ressonante é feita a

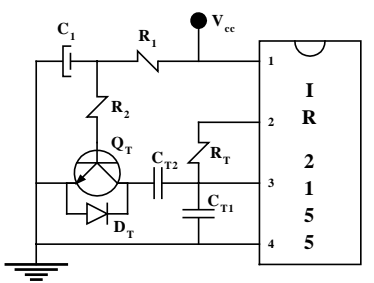

Figura 7 - Detalhe do circuito de controle projetado para propiciar o preaquecimento.

partir das equações (15), (16), (17) e (18).

$$
\begin{gathered}
\mathrm{C}_{\mathrm{s}}=\mathrm{G} \cdot \frac{\mathrm{P}_{\text {lamp }}}{\mathrm{V}_{\mathrm{AB}(\mathrm{ef})} \cdot \mathrm{V}_{\text {lamp(ef })}} \\
\mathrm{L}_{\mathrm{s}}=\frac{\mathrm{f}_{\mathrm{zvs}}^{2}}{\left(2 \cdot \pi \cdot \mathrm{f}_{\mathrm{HB}}\right)^{2} \cdot \mathrm{C}_{\mathrm{s}}} \\
\mathrm{C}_{\mathrm{p}}=\frac{\mathrm{f}_{\mathrm{ign}}^{2}}{\mathrm{f}_{\mathrm{zvs}}^{2}-\mathrm{f}_{\mathrm{ign}}^{2}} \cdot \mathrm{C}_{\mathrm{s}}
\end{gathered}
$$

sendo: $G=\frac{1-\mathrm{f}_{\mathrm{zvs}}^{2}}{2 . \pi \cdot \mathrm{f}_{\mathrm{HB}} \cdot \sqrt{1-\left(\frac{\mathrm{f}_{\mathrm{zvs}}^{2} \cdot\left(\mathrm{f}_{\mathrm{ign}}^{2}-1\right)}{\mathrm{f}_{\mathrm{zvs}}^{2}-\mathrm{f}_{\mathrm{ign}}^{2}} \cdot \frac{\mathrm{V}_{\text {lamp (ef })}}{\mathrm{V}_{\mathrm{AB}(\mathrm{ef})}}\right)^{2}}}$;

e: $\quad \mathrm{P}_{\mathrm{lamp}}=$ potência processada pela lâmpada fluorescente.

Destaca-se o fato de tais equações serem apresentadas de forma genérica, incorporando em sua formulação aspectos tais como o "batimento" e possibilitando maior precisão e flexibilidade ao cálculo dos parâmetros ressonantes, em relação a outras metodologias anteriormente propostas [7].

\section{EXEMPLO DE PROJETO}

Um exemplo de projeto do novo reator eletrônico é desenvolvido usando os dados de entrada e saída da Tabela I.

\section{A. Estágio Retificador Sepic ZCS-PWM com Elevado Fator de Potência}

O projeto do novo retificador Sepic ZCS-PWM é desenvolvido adotando-se os seguintes parâmetros:

$$
\beta=0,45 ; \mathrm{f}=0,14 \text { e } \alpha_{\text {máx }}=0,385 \text {. }
$$

Os elementos ressonantes da célula de comutação proposta são então determinados utilizando-se as equações (2), (3), (5) e (8), resultando em:

$$
\mathrm{C}_{\mathrm{r}}=13,2 \mathrm{nF} ; \mathrm{L}_{\mathrm{r} 1}=33 \mu \mathrm{H} \text { e } \mathrm{L}_{\mathrm{r} 2}=15 \mu \mathrm{H} \text {. }
$$

$O$ filtro de entrada $\left(\mathrm{L}_{\mathrm{in}}\right)$ é projetado de acordo com metodologia proposta em [5], resultando em:

$$
\mathrm{L}_{\mathrm{in}}=5 \mathrm{mH} \text {. }
$$


TABELA I

Dados de Entrada e Saída do Novo Reator Eletrônico

\begin{tabular}{|c|c|}
\hline Tensão eficaz de alimentação $\left(\mathrm{V}_{\text {in(ef }}\right)$ & $220 \mathrm{~V} \pm 15 \%$ \\
\hline Frequiência de chaveamento do retificador Sepic $\left(\mathrm{f}_{\text {Sepic }}\right)$ & $50 \mathrm{kHz}$ \\
\hline Valor médio da tensão no barramento $\mathrm{CC}\left(\mathrm{V}_{\mathrm{o}}\right)$ & $115 \mathrm{~V}$ \\
\hline $\begin{array}{c}\text { Freqüência de chaveamento do inversor Half-Bridge, } \\
\text { durante operação em regime }\left(\mathrm{f}_{\mathrm{HB}}\right)\end{array}$ & $50 \mathrm{kHz}$ \\
\hline Valor eficaz da tensão sobre a lâmpada $\left(\mathrm{V}_{\text {lamp(eff }}\right)$ & $120 \mathrm{~V}$ \\
\hline $\begin{array}{c}\text { Freqüência de chaveamento do inversor Half-Bridge, } \\
\text { durante o processo de preaquecimento }\left(\mathrm{f}_{\mathrm{HB}(\mathrm{ph})}\right)\end{array}$ & $85 \mathrm{kHz}$ \\
\hline Intervalo de tempo destinado ao preaquecimento & $150 \mathrm{~ms}$ \\
\hline Valor "pico-a-pico" da tensão de ignição da lâmpada & $500 \mathrm{~V}$ \\
\hline Mínimo rendimento do estágio retificador $(\eta \%)$ & $95 \%$ \\
\hline Potência nominal de saída $\left(\mathrm{P}_{\mathrm{o}}\right)$ & $200 \mathrm{~W}$ \\
\hline
\end{tabular}

A indutância de acumulação $\left(\mathrm{L}_{\mathrm{M}}\right)$ é obtida para uma ondulação de corrente inferior a $20 \%$ do valor nominal de corrente processada. A determinação da capacitância de acumulação $\left(\mathrm{C}_{\mathrm{e}}\right)$ é um compromisso entre a necessidade de reduzida ondulação de tensão em elevada freqüência e reduzida TDH na corrente de entrada. Portanto, são especificados:

$$
\mathrm{L}_{\mathrm{M}}=2 \mathrm{mH} \text { e } \mathrm{C}_{\mathrm{e}}=330 \mathrm{nF} \text {. }
$$

$\mathrm{O}$ filtro de saída $\left(\mathrm{C}_{\mathrm{o}}\right)$ é especificado assumindo que a ondulação da tensão sobre o barramento $\mathrm{CC}$ deve ser restringida a $2 \%$ do valor nominal de $\mathrm{V}_{\mathrm{o}}$. Assim sendo:

$$
\mathrm{C}_{\mathrm{o}}=1360 \mu \mathrm{F} \text {. }
$$

Os semicondutores de potência utilizados no protótipo laboratorial foram:

- Interruptores: $\mathrm{S}_{1}: 12 \mathrm{~N} 60 \mathrm{~A} 4 \mathrm{D}$ (IGBT);

$$
\mathrm{S}_{2}: 7 \mathrm{~N} 60 \mathrm{~A} 4 \mathrm{D} \text { (IGBT). }
$$

- diodos: $\mathrm{D}_{1}$ : MUR8100E (diodo ultra-rápido); $\mathrm{D}_{2}$ : MUR8100E (diodo ultra-rápido).

Por fim, o projeto do controle por valores médios da corrente de entrada é realizado de acordo com metodologia apresentada em [11]

\section{B. Estágio Inversor Ressonante Half-Bridge}

É assumido que o processo de preaquecimento deverá ocorrer no mínimo durante $150 \mathrm{~ms}$, fato que implica na escolha dos seguintes componentes:

$$
\mathrm{R}_{1}=47 \mathrm{k} \Omega ; \mathrm{R}_{2}=1,2 \mathrm{k} \Omega \text { e } \mathrm{C}_{1}=220 \mu \mathrm{F} .
$$

Adota-se ainda que a frequiência de chaveamento a ser imposta durante o preaquecimento seja de $85 \mathrm{kHz}$.

Desta forma, para a imposição das devidas freqüências de chaveamento, os seguintes parâmetros são adotados:

$$
\mathrm{R}_{\mathrm{T}}=1635 \mathrm{k} \Omega ; \mathrm{C}_{\mathrm{T} 1}=4,7 \mathrm{nF} \text { e } \mathrm{C}_{\mathrm{T} 2}=3,3 \mathrm{nF} \text {. }
$$

Em caso de falha no processo de ignição das lâmpadas, o valor máximo de tensão sobre as mesmas será limitado a $1500 \mathrm{~V}$, através da adoção de um valor adequado para $\mathrm{f}_{\mathrm{ign}}$. Além disso, a comutação ZVS em $\mathrm{S}_{3}$ e $\mathrm{S}_{4}$ deve ser garantida através da adoção de $\mathrm{f}_{\mathrm{zvs}}$ em um valor superior àquele determinado através da equação (14). Portanto adota-se:

$$
f_{\text {ign }}=1,075 \text { e } f_{\mathrm{zvs}}=4 \text {. }
$$

Em função de tais valores, os elementos ressonantes especificados para o inversor Half-Bridge são:

$$
\begin{gathered}
\mathrm{C}_{\mathrm{s}}=\mathrm{C}_{\mathrm{s} 1} \text { até } \mathrm{C}_{\mathrm{s} 5}=330 \mathrm{nF} ; \\
\mathrm{L}_{\mathrm{s}}=\mathrm{L}_{\mathrm{s} 1} \text { até } \mathrm{L}_{\mathrm{s} 5}=500 \mu \mathrm{F} ; \mathrm{e} \\
\mathrm{C}_{\mathrm{p}}=\mathrm{C}_{\mathrm{p} 1} \text { até } \mathrm{C}_{\mathrm{p} 5}=22 \mathrm{nF} \text {. }
\end{gathered}
$$

Os semicondutores de potência utilizados foram:

$$
\text { Interruptores: } \mathrm{S}_{3} \text { e } \mathrm{S}_{4} \text { : IRF740 (MOSFET). }
$$

\section{RESULTADOS EXPERIMENTAIS}

A fotografia de um protótipo do novo reator eletrônico com elevado fator de potência é mostrada na Figura 8.

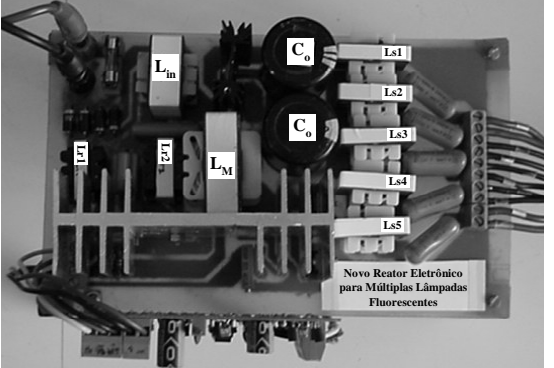

Figura 8 - Protótipo do novo reator eletrônico implementado.

A Figura 9.a mostra a forma de onda da corrente de entrada do reator, para uma tensão de alimentação nominal e operação em plena carga. Com base nesta figura, é possível verificar que a defasagem angular entre a corrente de entrada e a tensão de alimentação é desprezível. Para carga nominal, o espectro de frequiências da corrente de entrada é mostrado na Figura 9.b e sua TDH é igual a 7,59\%, para uma TDH na tensão de alimentação igual a $1,56 \%$. O fator de potência da estrutura nesta condição é de aproximadamente 0,986 .

Os valores das principais componentes harmônicas das correntes de entrada, obtidos através de análise da forma de onda apresentada na Figura 9, são mostrados na Tabela II. Nesta tabela, são também apresentados os valores máximos permitidos pela norma IEC 61000-3-2 para equipamentos classe $\mathrm{C}$, referentes a dispositivos destinados a iluminação. Com base na Tabela II, é possível verificar que o novo reator eletrônico enquadra-se em todas as exigências da norma IEC 61000-3-2 para equipamentos classe $\mathrm{C}$.

A Figura 10 mostra os detalhes das comutações dos interruptores usados no estágio retificador Sepic. Estas formas de onda foram obtidas para a situação em que o valor instantâneo da tensão de alimentação é próximo de zero $\left(\mathrm{V}_{\text {in }}(\omega \mathrm{t}) \cong 0\right)$ e próximo ao valor de pico $\left(\mathrm{V}_{\text {in }}(\omega \mathrm{t}) \cong \mathrm{V}_{\text {in(p) }}\right)$.

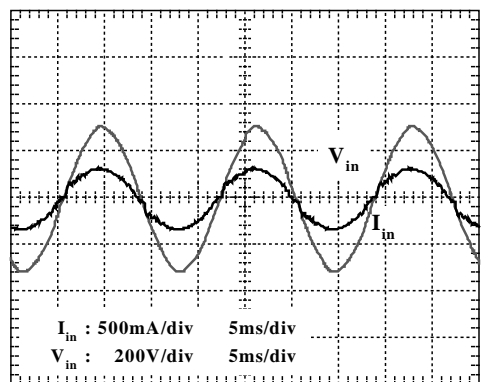

(a)

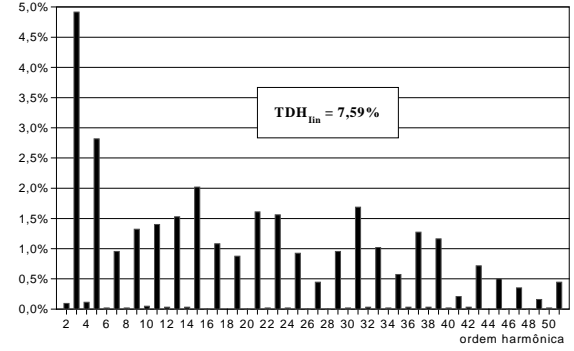

(b)

Figura 9 - (a) Tensão de alimentação e corrente de entrada, e (b) espectro de freqüências de $\mathrm{I}_{\text {in }}$, para carga nominal. 


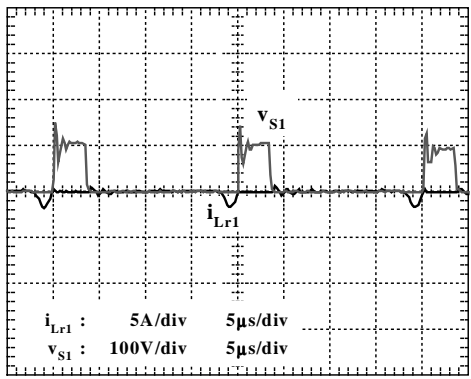

(a) $\mathrm{V}_{\text {in }}(\mathrm{t}) \cong 0$

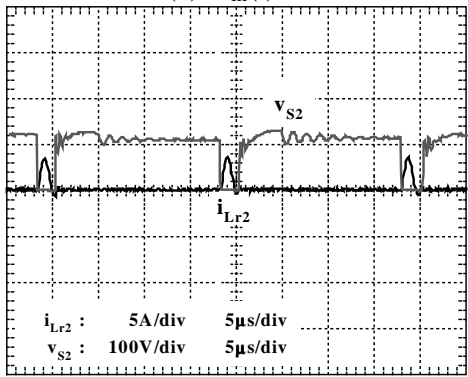

(c) $\mathrm{V}_{\text {in }}(\mathrm{t}) \cong 0$

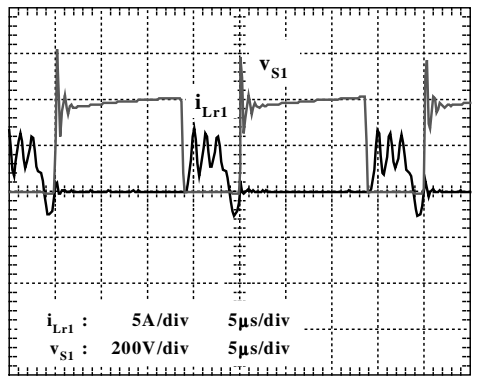

(b) $\mathrm{V}_{\text {in }}(\mathrm{t}) \cong \mathrm{V}_{\text {in( } \mathrm{p})}$

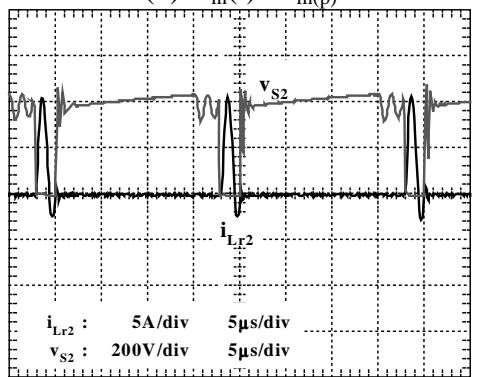

(d) $\mathrm{V}_{\text {in }}(\mathrm{t}) \cong \mathrm{V}_{\text {in }(\mathrm{p})}$

Figura 10 - Detalhes de comutações de $S_{1}$, para carga nominal: (a) próximo a $V_{\text {in }}(t)=0$, e (b) próximo a $V_{\text {in }}(t)=V_{\text {in( }(p)}$; Detalhes de comutações de $S_{2}$, para carga nominal: (c) próximo a $V_{\text {in }}(t)=0$, e (d) próximo a $V_{\text {in }}(t)=V_{\text {in }(p)}$.

TABELA II

Comparação entre a norma IEC 61000-3-2 para equipamentos classe $\mathrm{C}$ e componentes harmônicas da corrente de entrada, medidas a plena carga

\begin{tabular}{|c|c|c|}
\hline $\begin{array}{c}\text { Ordem } \\
\text { Harmônica }\end{array}$ & $\begin{array}{c}\text { IEC 61000-3-2 } \\
\text { Máxima Corrente } \\
\text { Harmônica Aceitável } \\
\text { ([\%] da componente } \\
\text { fundamental da } \\
\text { corrente de entrada) }\end{array}$ & $\begin{array}{c}\text { Corrente Harmônica Medida } \\
\text { ([\%] da componente } \\
\text { fundamental da } \\
\text { corrente de entrada) }\end{array}$ \\
\hline 2 & 2 & 0,12 \\
\hline 3 & $30 . \lambda\left(^{*}\right)=29,58$ & 4,76 \\
\hline 5 & 10 & 2,73 \\
\hline 7 & 7 & 0,95 \\
\hline 9 & 5 & 1,30 \\
\hline $11 \leq \mathrm{n} \leq 39$ & 3 & $<<3$ \\
\hline \multicolumn{2}{|c|}{} & $(*) \lambda$ é o fator de potência do circuito \\
\hline
\end{tabular}

De acordo com a Figura 10, pode-se notar que $S_{1}$ e $S_{2}$ apresentam entrada em condução do tipo ZCS e bloqueio do tipo ZCZVS. É importante observar que estas comutações suaves são preservadas durante todo o período da rede de alimentação em CA, implicando na obtenção de elevado rendimento para este estágio.

Finalmente, é importante informar que neste novo arranjo para a célula de comutação suave, os esforços de tensão sobre os interruptores $S_{1}$ e $S_{2}$ são muito menores do que aqueles verificados em [5].

Detalhes das comutações dos interruptores $\mathrm{S}_{3}$ e $\mathrm{S}_{4}$, do estágio inversor Half-Bridge, são mostrados na Figura 11. A partir desta figura, pode-se notar que a entrada em condução de ambos os interruptores ocorre de forma ZVS. O rendimento global medido para este novo reator eletrônico é de aproximadamente $92,1 \%$ para operação em plena carga.

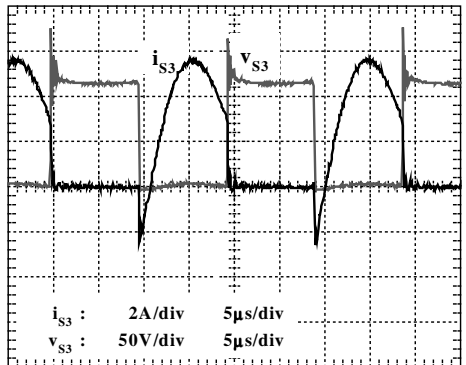

(a)

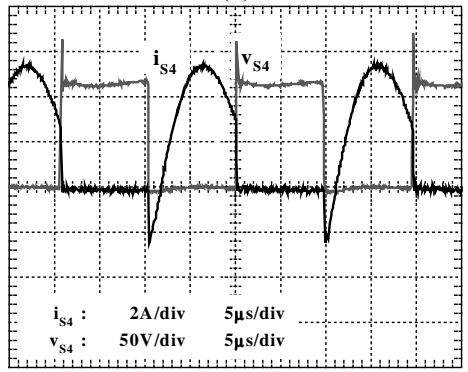

(b)

Figure 11 - Detalhes de comutações no inversor Half-Bridge, para carga nominal: (a) interruptor $S_{3}$, e (b) interruptor $S_{4}$.

A tensão sobre uma das lâmpadas e a corrente através do respectivo circuito oscilador para a operação em regime são mostradas na Figura 12.a, em conjunto com detalhes do processo de ignição mostrados na Figuras 12.b e 12.c.

Foi verificado que o fator de crista desta estrutura proposta é de aproximadamente 1,42. Com base na Figura 12.b, é possível verificar que o preaquecimento ocorre em um intervalo de tempo de aproximadamente 200ms. Durante este intervalo de tempo, a tensão sobre a lâmpada fluorescente evolui através de duas etapas até atingir o valor requerido para proporcionar a ignição da lâmpada. Da Figura 12.c, pode-se notar que o máximo valor de tensão sobre a lâmpada é limitado pela ocorrência do "batimento", como 
esperado.

Desta forma, em caso de falha do processo de ignição de qualquer uma das lâmpadas, não ocorrerão danos aos componentes deste novo reator eletrônico em função de esforços de tensão e corrente demasiados.

\section{CONCLUSÕES}

Este artigo apresentou um novo reator eletrônico para múltiplas lâmpadas fluorescentes tubulares.

O estágio de entrada deste novo reator eletrônico é um retificador Sepic ZCS-PWM com elevado fator de potência. Os interruptores ativos $S_{1}$ e $S_{2}$ desta topologia apresentam entrada em condução do tipo ZCS e bloqueio do tipo ZCZVS. Informa-se que os diodos $\mathrm{D}_{1}$ e $\mathrm{D}_{2}$ apresentam entrada em condução do tipo ZVS e seus efeitos de recuperação reversa sobre os interruptores ativos são reduzidos. Este novo arranjo da célula de comutação suave proporciona a obtenção de reduzidos esforços de tensão sobre os interruptores ativos, quando comparados àqueles obtidos na célula de comutação original [5], além do que, $\mathrm{D}_{1}$ e $\mathrm{D}_{2}$ não se associam em série, melhorando o rendimento do estágio de entrada. Utilizando-se o controle por valores médios instantâneos de corrente para o estágio retificador, foi possível a obtenção de reduzida TDH na corrente de entrada, além de reduzida defasagem angular entre a corrente de entrada e a tensão de alimentação, fatos estes que implicam em um conseqüente elevado fator de potência. Os resultados obtidos a partir do protótipo implementado para este novo reator eletrônico encontram-se em concordância com as normas IEC 61000-3-2 para equipamentos classe C.

Com relação ao estágio de saída, pode-se concluir que os interruptores ativos $S_{3}$ e $S_{4}$ apresentam entrada em condução do tipo ZVS, conforme esperado. A eliminação das perdas associadas aos processos de comutação dos estágios retificador e inversor assegura a obtenção de elevada eficiência global $(92,1 \%$, para carga nominal).

Por fim, com relação ao estágio de saída, foi desenvolvida uma metodologia adequada para o projeto do circuito inversor Half-Bridge, incluindo-se a previsão de ocorrência do fenômeno conhecido como "batimento", no procedimento de cálculo dos elementos ressonantes, possibilitando o uso de um integrado de baixo custo para alimentação de cinco lâmpadas fluorescentes.

\section{AGRADECIMENTOS}

Os autores agradecem à FAPESP pelo apoio concedido ao desenvolvimento deste trabalho.

\section{REFERÊNCIAS BIBLIOGRÁFICAS}

[1] M. S. Rea, The IESNA Lighting Handbook - Reference and Application, Illuminating Engineering Society of North America, 9 $9^{\mathrm{a}}$ Edição, em CD-ROM.

[2] J. Spangler, B. Hussain, e A. K. Behera, "Electronic Fluorescent Ballast using Power Factor Correction Techniques for Loads greater than 300Watts", Anais do IEEE APEC - Applied Power Electronics Conference, 1991, pp. 393-399.

[3] R. Gules, E. U. Simões, e I. Barbi, "A $1.2 \mathrm{~kW}$
Electronic Ballast for Multiple Lamps, with Dimming Capability and High Power-Factor", Anais do IEEE APEC - Applied Power Electronics Conference, 1999, pp. 720-726.

[4] F. T. Wakabayashi, M. J. Bonato, e C. A. Canesin, "Novel High-Power-Factor ZCS-PWM Preregulators", IEEE Transactions on Industrial Electronics, vol. 48, n⿳⺈ 2, pp. 322-333, Abril 2001.

[5] L. T. S. Sobrinho, R. A. Kitamura e C. A. Canesin, "Novel Zero-Current-Switching PWM Step-Down Sepic Converter", Anais do PEDES - Conference on Power Electronics Drive and Energy Systems for Industrial Growth, 1998, pp. 717-722.

[6] E. E. Hammer, "High Frequency Characteristics of Fluorescent Lamps up to $500 \mathrm{kHz}$ ", Journal of IES, winter 1987, pp.52-61.

[7] A. S. André, M. V. A. Araújo, A. J. Perin, e I. Barbi, "Reator Eletrônico Auto-Oscilante para Duas Lâmpadas Fluorescentes de 65W", Anais do CBA Congresso Brasileiro de Automática, 2000, em CDROM.

[8] A. S. André, e A. J. Perin, "Reator Eletrônico para lâmpadas fluorescentes de $40 \mathrm{~W}$ utilizando Circuito Integrado Dedicado", Anais do SEP - Seminário de Eletrônica de Potência (UFSC), Florianópolis (SC), 1998.

[9] J. Parry, "Variable Frequency Drive using IR215X Self Oscillating IC's", Lighting Ballast control IC Designer's Manual 2001, International Rectifier, pp.331-338.

[10] "IR2155 - Self-Oscillating Half-Bridge Driver", Data Sheet $n^{-}$PD60029-I, International Rectifier.

[11] P. C. Todd, "UC3854 Controlled Power Factor Correction Circuit Design", Application note U-134, Unitrode.

\section{DADOS BIOGRÁFICOS}

Fabio Toshiaki Wakabayashi, nascido em Jales (SP), em Julho de 1974, é engenheiro eletricista (1996) e mestre em Engenharia Elétrica (1998), formado na Universidade Estadual Paulista - Faculdade de Engenharia de Ilha Solteira (UNESP-FEIS, Ilha Solteira (SP)), onde atualmente desenvolve o doutorado em Eletrônica de Potência. Suas áreas de interesse abrangem técnicas de comutação nãodissipativa, fontes de alimentação chaveadas, qualidade de energia elétrica e reatores eletrônicos para iluminação.

Carlos Alberto Canesin, nascido em Lavínia (SP), em 1961, é engenheiro eletricista (1985) pela Universidade Estadual Paulista - Faculdade de Engenharia de Ilha Solteira (UNESP-FEIS, Ilha Solteira (SP)), mestre (1990) e doutor (1996) em Engenharia Elétrica pela Universidade Federal de Santa Catarina - Instituto de Eletrônica de Potência (UFSCINEP), Florianópolis (SC). Atualmente é professor adjunto efetivo do Departamento de Engenharia Elétrica (DEE) da UNESP-FEIS. Suas áreas de interesse incluem técnicas de comutação não-dissipativa, conversores $\mathrm{CC} / \mathrm{CC}$, fontes de alimentação chaveadas, reatores para iluminação e técnicas de correção do fator de potência. 


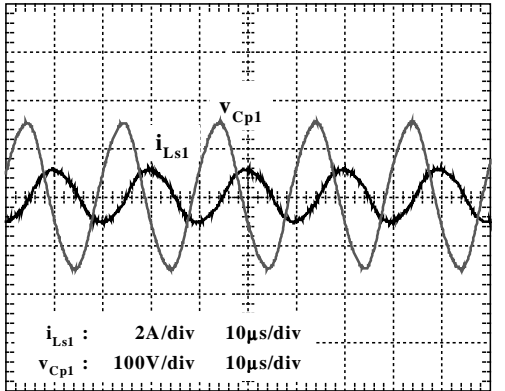

(a)

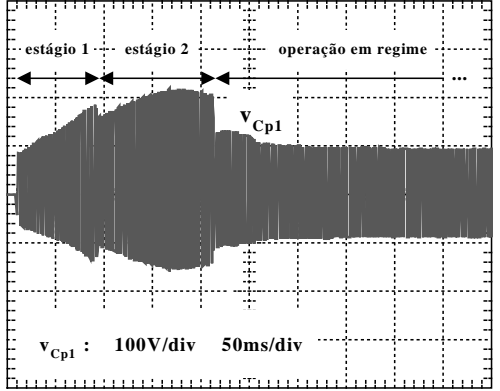

(b)

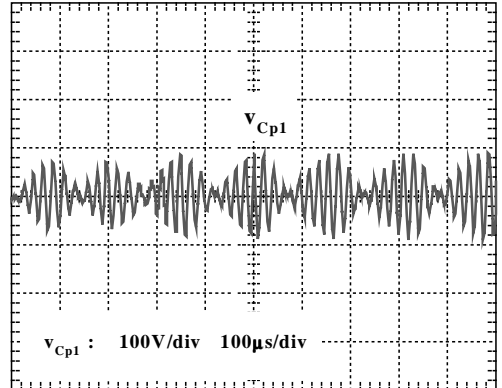

(c)

Figura 12 - (a) Tensão sobre uma das lâmpadas e corrente através de um dos circuitos osciladores; Detalhes de ignição de uma das lâmpadas: (b) tempo de preaquecimento, e (c) "batimento". 